\title{
Structure and stability of a homologous series of tin oxides
}

$\operatorname{AUTHOR}(\mathrm{S}):$

Seko, Atsuto; Togo, Atsushi; Oba, Fumiyasu;

Tanaka, Isao

\section{CITATION:}

Seko, Atsuto ...[et al]. Structure and stability of a homologous series of tin oxides. Physical Review Letters 2008, 100(4): 045702.

\section{ISSUE DATE:}

2008-02-01

URL:

http://hdl.handle.net/2433/84641

RIGHT:

(C) 2008 The American Physical Society 


\title{
Structure and Stability of a Homologous Series of Tin Oxides
}

\author{
Atsuto Seko, ${ }^{1, *}$ Atsushi Togo, ${ }^{2, \dagger}$ Fumiyasu Oba, ${ }^{2}$ and Isao Tanaka ${ }^{2,3}$ \\ ${ }^{1}$ Pioneering Research Unit for Next Generation, Kyoto University, Kyoto 615-8530, Japan \\ ${ }^{2}$ Department of Materials Science and Engineering, Kyoto University, Kyoto 606-8501, Japan \\ ${ }^{3}$ Nanostructure Research Laboratory, Japan Fine Ceramics Center, Atsuta, Nagoya, 456-8587 Japan
}

(Received 25 August 2007; published 30 January 2008)

\begin{abstract}
The structures and stabilities of a series of nonstoichiometric $\mathrm{SnO}_{2-x}$ compounds, which are yet unknown experimentally, are predicted using the cluster expansion technique combined with firstprinciples calculations. A homologous series of $\mathrm{Sn}_{n+1} \mathrm{O}_{2 n}$ in which oxygen vacancies are layered on (101) planes of the rutile lattice is discovered. The homologous crystals are composed of divalent and quadrivalent $\mathrm{Sn}$ atoms. No trivalent $\mathrm{Sn}$ atoms are formed.
\end{abstract}

DOI: 10.1103/PhysRevLett.100.045702

The oxides with quadrivalent cations often form a rutiletype structure. Two minerals with this structure known as rutile $\left(\mathrm{TiO}_{2}\right)$ and cassiterite $\left(\mathrm{SnO}_{2}\right)$ have been popular subjects of fundamental studies for over a century. These oxides are also important in modern technology as catalysts and in spintronic and optoelectronic applications. Both of them often show oxygen deficiency, which plays a central role in determining their properties and chemical activities. In the Ti-O system, a series of nonstoichiometric compounds or homologous compounds are known to exist and are called "Magnéli phase" [1-3]. Many studies have also been carried out on the nonstoichiometric compounds of $\mathrm{SnO}_{2-x}$ [4-14]. However, the structures and stabilities are much less understood. Although several phase diagrams have been reported for the Sn-O binary system, they do not agree with each other [4,5]. Even the stability of the monoxide, $\mathrm{SnO}$, has not been well established. Some studies have reported that $\mathrm{SnO}$ is a stable phase at a temperature range from $298 \mathrm{~K}$ to $543 \mathrm{~K}$ [4]. Another group did not show $\mathrm{SnO}$ in their equilibrium phase diagram [5]. The structures and stabilities of intermediate compounds, such as $\mathrm{Sn}_{2} \mathrm{O}_{3}, \mathrm{Sn}_{3} \mathrm{O}_{4}$, and $\mathrm{Sn}_{5} \mathrm{O}_{6}$, are still open to debate. Recently, possible structures of $\mathrm{Sn}_{2} \mathrm{O}_{3}$ were discussed theoretically using first-principles calculations through the examination of eight candidate structures [8]. However, their selection of the structures is far from complete and thus is not persuasive. Structures should be searched for within much larger degrees of freedom. For the other nonstoichiometric $\mathrm{SnO}_{2-x}$ compounds, no firstprinciples studies have thus far been reported. To determine the unknown structures, a theoretical approach combining first-principles calculations with a cluster expansion technique [15-17] can be a powerful tool. In the present study, a systematic study of the nonstoichiometric compounds in the Sn-O binary system is carried out by the combined approach.

In general, nonstoichiometry originates from various kinds of point defects. They often form extended structures. To evaluate the thermodynamics of the nonstoichiometric structures, the formation energies of all different defects and their extended structures should be known in
PACS numbers: 81.30.Dz, 61.50.Ah, 61.66.Fn, 61.72.Bb

principle. In the practical level, an appropriate modeling of the defective structures is essential for experimentally structure-unknown systems. In the present case of the Sn$\mathrm{O}$ system, no experimental structures have been reported except for $\mathrm{SnO}$ and $\mathrm{SnO}_{2}$. $\mathrm{SnO}$ and $\mathrm{SnO}_{2}$ have litharge $(\alpha-\mathrm{PbO})$ and rutile structures, as shown in Fig. 1(a). Figure 1(b) illustrates the projections of the structures of $\mathrm{SnO}$ and $\mathrm{SnO}_{2}$ to the (010) plane. They have common features although their space group and the fractional positions of oxygen atoms are different. Tin atoms form a body-centered-tetragonal sublattice in both $\mathrm{SnO}$ and $\mathrm{SnO}_{2}$. Six oxygen atoms are coordinated to each tin atom in $\mathrm{SnO}_{2}$. In $\mathrm{SnO}$, half the oxygen atoms are removed from rutile $\mathrm{SnO}_{2}$, as shown in Fig. 1(b). The other oxygen atoms are asymmetrically coordinated to the tin atoms. From the inspection of these structures, we have adopted the oxygen sublattice model for the intermediate structures in which $\mathrm{SnO}_{2-x}(0<x<1)$ is described by the arrangements of oxygen atoms and vacancies on the oxygen sublattice of the rutile $\mathrm{SnO}_{2}$. We have also examined the formation energies of a series of nonstoichiometric $\mathrm{SnO}_{2-x}$ compounds using the well-known $\mathrm{TiO}_{2-x}$ Magnéli structures. The formation energy of the Magnéli structure for $\mathrm{Sn}_{4} \mathrm{O}_{7}$ is found to be more than $+400 \mathrm{meV} /$ cation with reference to $\mathrm{SnO}$ (litharge) and $\mathrm{SnO}_{2}$ (rutile), which is prohibitively high. This implies that the origin of the nonstoichiometry (a)

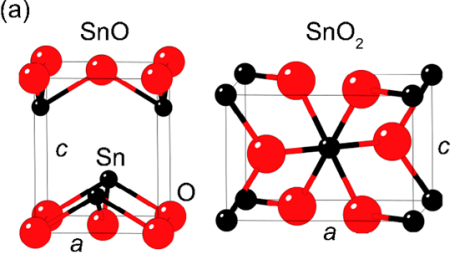

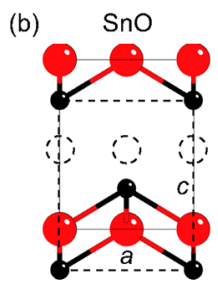

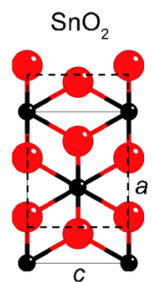

FIG. 1 (color online). (a) Structures of litharge $\mathrm{SnO}$ and rutile $\mathrm{SnO}_{2}$. Both $\mathrm{SnO}$ and $\mathrm{SnO}_{2}$ show tetragonal symmetry. Experimental lattice constants of $\mathrm{SnO}$ and $\mathrm{SnO}_{2}$ are $a=$ $3.850 \AA, c=4.901 \AA$ and $a=4.738 \AA, c=3.188 \AA$, respectively. (b) Projections of the structures to (010) plane. The broken circles in $\mathrm{SnO}$ correspond to removed oxygen atoms from rutile $\mathrm{SnO}_{2}$. 
or the multivalency in the Sn-O system is different from that in the Ti-O system although both $\mathrm{TiO}_{2}$ and $\mathrm{SnO}_{2}$ have the rutile structure.

After determining the model used to describe the configurational energetics, first-principles total energies of structures in which atoms are arranged in accordance with the model are required to construct the effective Hamiltonian. We need to describe the precise configurational energetics of the pseudobinary oxygen-vacancy system in the oxygen sublattice to investigate the phase stability of the nonstoichiometric structures. It is, however, practically impossible to calculate configurational energies from first principles for all possible structures due to computational costs. A combination of first-principles calculations and a cluster expansion, which provides an effective configurational Hamiltonian in multicomponent systems, enables us to overcome this difficulty. Within the cluster expansion formalism, the configurational energy $E$ can be expressed as

$$
E=V_{0}+\sum_{i} V_{i} \sigma_{i}+\sum_{i, j} V_{i j} \sigma_{i} \sigma_{j}+\sum_{i, j, k} V_{i j k} \sigma_{i} \sigma_{j} \sigma_{k}+\cdots,
$$

where the coefficients $V$ are called effective cluster interaction (ECI) and $\sigma_{i}$ denotes the pseudospin configuration variable for the respective lattice site $i$; if the lattice site $i$ is occupied by an oxygen atom (vacancy), then $\sigma_{i}=1$ ( $\sigma_{i}=$ $-1)$. The values of ECI were determined from the set of first-principles total energies of ordered structures using a least-squares method. The number of structures usually ranges 30-100 to estimate ground states and phase diagrams in close-packed systems. In the Sn-O system, more structures to calculate energies from first principles are indispensable in order to obtain more ECIs than those in the closed-packed systems. This is because the rutile structure has lower symmetry than the close-packed lattice and the effects of lattice relaxations are large as expected from the large difference in the lattice constants between $\mathrm{SnO}$ and $\mathrm{SnO}_{2}$. We calculated the total energies for 750 ordered structures in which oxygen atoms and vacancies are placed on the oxygen sublattice. These structures include an initial set of random structures and some lower energy structures iteratively refined through the cluster expansion. The computed structures have various numbers of atoms ranging up to 48 atoms. For the calculation of the total energies, we used the projector augmented-wave method [18] within the generalized gradient approximation [19] as implemented in the VASP code [20-22]. Sn- $5 s, 5 p$ and O-2s, $2 p$ were treated as valence. The plane-wave cutoff energy was set at $300 \mathrm{eV}$. The total energies were converged to less than $10^{-5} \mathrm{eV}$. The atomic positions and lattice constants were relaxed until the residual forces became less than $0.01 \mathrm{eV} / \AA$. A set of ECI used to construct the effective Hamiltonian were optimized using a genetic algorithm (GA) [23], which is an evolutionary algorithm used to solve optimization problems. The set of ECI minimizes the cross-validation score (CV) [24,25], which can determine the accuracy of the cluster expansion that a selected set of ECI reproduces the physical properties well. We eventually selected 28 clusters consisting of the empty and point clusters, 13 pairs, 9 triplets, and 4 quadruplets from 262 clusters up to quadruplets using GA. The corresponding CV was $18.2 \mathrm{meV} /$ anion site. Once the optimal set of ECI is known and the effective Hamiltonian is constructed, one can predict the stable phases and all the configuration-dependent thermodynamical properties on the basis of statistical mechanics. We used the CLUPAN code [26-28] for a series of calculations such as optimization of clusters and the successive searches for stable phases.

There are two kinds of approaches that can be used to find the stable structures using the obtained effective Hamiltonian. One is an exhaustive search by calculating the energies for all possible configurations in a finite-sized cell. This is the most precise way to search for stable structures. However, the space of the search is obviously limited by computational resources. An alternative way is to use some efficient algorithms to solve optimization problems such as simulated annealing (SA) [29,30]. A much larger space of structures can be searched using larger supercells than that by the exhaustive search. Figure 2(a) shows the calculated formation energies relative to the energies of litharge $\mathrm{SnO}$ and rutile $\mathrm{SnO}_{2}$ using the effective Hamiltonian for all configurations within seven different kinds of supercells that are composed of less than 32 oxygen sites. Several structures show singularly low energies. The structure having the lowest energy for $\mathrm{Sn}_{2} \mathrm{O}_{3}(x=0.5)$ is illustrated in Fig. 2(b). The other low-energy structures have similar features to this structure with oxygen vacancies stacked on the (100) layer. They consist of $\mathrm{SnO}_{2}$-like local structures including $\mathrm{SnO}_{6}$ octahedrons and SnO-like local structures in which $\mathrm{Sn}$ atoms face each other. We also performed SA using larger super-

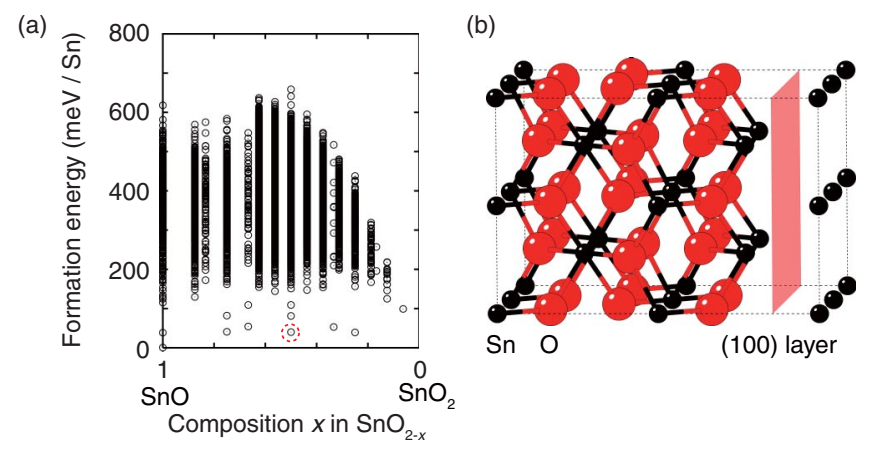

FIG. 2 (color online). (a) Calculated formation energies using the effective Hamiltonian for all structures in which oxygen atoms and vacancies are placed on seven different kinds of supercells that are composed of less than 32 oxygen sites. (b) Structure having the lowest energy for $\mathrm{Sn}_{2} \mathrm{O}_{3}$ viewed from near the [010] direction. The corresponding energy is shown by the broken circle in (a). Oxygen vacancies forming a (100) layer in the structure are shown by the quadrangle. 


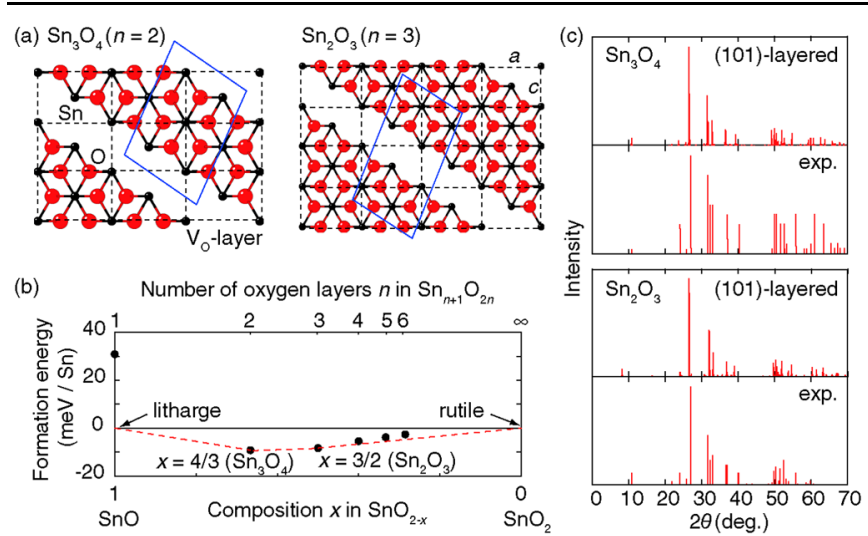

FIG. 3 (color online). (a) Predicted stable structures for $\mathrm{Sn}_{3} \mathrm{O}_{4}$ and $\mathrm{Sn}_{2} \mathrm{O}_{3}$ by simulated annealing. The blue solid quadrilaterals show unit cells. (b) Formation energies of (101)-layered structures, shown by closed circles, relative to litharge $\mathrm{SnO}$ and rutile $\mathrm{SnO}_{2}$. The closed circle at $x=1(\mathrm{SnO})$ indicates the formation energy of the hypothetical (101)-layered structure for $\mathrm{SnO}$ compound. The broken line represents the convex hull of the ground state. (c) Theoretical XRD patterns $\left(\mathrm{Cu}-K_{\alpha}\right)$ of the predicted structures for $\mathrm{Sn}_{3} \mathrm{O}_{4}$ and $\mathrm{Sn}_{2} \mathrm{O}_{3}$ compared with the experimental XRD patterns reported in Refs. $[9,10]$, respectively.

cells up to $12 \times 12 \times 12$ (10368 atoms). More stable structures for $\mathrm{Sn}_{3} \mathrm{O}_{4}$ and $\mathrm{Sn}_{2} \mathrm{O}_{3}$ are found by $\mathrm{SA}$ as shown in Fig. 3(a). The structural parameters and space groups are listed in Table I. In the predicted structures, oxygen vacancies are aligned along the plane corresponding to (101) of $\mathrm{SnO}_{2}$. They are composed of $\mathrm{SnO}_{2}$-like and SnO-like local structures. Similar structures for the other oxygen-deficient compounds using various sizes of supercells were also predicted by SA. The (101)-layered structures can be expressed as a series of homologous structures of $\mathrm{Sn}_{n+1} \mathrm{O}_{2 n}$ with alternating $n$ layers of $\mathrm{SnO}_{2}$-like bands and a (101) vacancy layer. Mäki-Jaskari et al. calculated the energies for eight possible crystals and interfaces for $\mathrm{Sn}_{2} \mathrm{O}_{3}$ [8]. Our extensive exploration of the stable structures coincides with their conclusion that the most stable one is the (101)-layered crystal. To discuss the relative stability of $\mathrm{Sn}_{n+1} \mathrm{O}_{2 n}$, it is preferable to use first-principles energies because the magnitudes of energy differences among $\mathrm{Sn}_{n+1} \mathrm{O}_{2 n}$ with the order of a few millielectron volts per $\mathrm{Sn}$ atom are too small to be accurately evaluated using the effective Hamiltonian. Figure 3(b) shows the formation energies of the (101)-layered structures obtained from first principles. Other than $n=1$, the formation energies are negative. Both $\mathrm{Sn}_{3} \mathrm{O}_{4}(n=2)$ and $\mathrm{Sn}_{2} \mathrm{O}_{3}(n=3)$ are stable, and the other (101)-layered structures ( $n=1,4-$ 6) are metastable. However, considering the tiny energy differences among $\mathrm{Sn}_{n+1} \mathrm{O}_{2 n}(n=2-6)$, the metastable structures may also be observed by experiments.

Although the structures of $\mathrm{SnO}_{2-x}$ compounds have not been determined experimentally, x-ray diffraction (XRD) patterns of two possible compounds have been reported for the compositions of $\mathrm{Sn}_{3} \mathrm{O}_{4}$ and $\mathrm{Sn}_{2} \mathrm{O}_{3}[9,10]$. Figure 3(c) shows the theoretical XRD patterns of the stable structures for $\mathrm{Sn}_{3} \mathrm{O}_{4}$ and $\mathrm{Sn}_{2} \mathrm{O}_{3}$ compared with the experimental powder XRD patterns. The theoretical XRD patterns for the predicted (101)-layered structure of $\mathrm{Sn}_{3} \mathrm{O}_{4}$ and $\mathrm{Sn}_{2} \mathrm{O}_{3}$ are very close to each other, reflecting the similarities of the structures based on the common $\mathrm{SnO}$ and $\mathrm{SnO}_{2}$-like units. The peak positions and relative intensities of the theoretical XRD patterns are close to those of the experimental XRD patterns [31]. The agreements of the theoretical and experimental XRD patterns indicate that the $\mathrm{Sn}_{3} \mathrm{O}_{4}$ and $\mathrm{Sn}_{2} \mathrm{O}_{3}$ phases observed experimentally in tin oxide systems correspond to the (101)-layered structures based on the mother rutile structure. Recently transmission electron microscope observation of $\mathrm{SnO}_{2-x}$ thin film was reported [7]. Nonstoichiometric $\mathrm{SnO}_{2-x}$ was found to be formed during annealing a $\mathrm{SnO}$ thin film in air before it eventually transformed to the rutile-type $\mathrm{SnO}_{2}$. The $\mathrm{SnO}_{2-x}$ film showed a number of planer faults that are parallel to the $\mathrm{SnO}_{2}$ (101) plane. The formation of the faults was ascribed to the presence of oxygen deficiency. Although information of atomic structures was not reported in the Letter, the formation of (101)-type planer faults in the nonstoichiometric composition is consistent with the structure predicted in the present study.

The electronic structures of the homologous phases are investigated together with two reference phases, $\mathrm{SnO}_{2}$ and $\mathrm{SnO}$. They can be most clearly compared with the projected density of states (PDOS) of Sn atoms. Figure 4 shows the PDOS of two kinds of $\mathrm{Sn}$ atoms in $\mathrm{Sn}_{2} \mathrm{O}_{3}$ along with the PDOS of $\mathrm{Sn}$ atoms in $\mathrm{SnO}_{2}$ and $\mathrm{SnO}$. The $\mathrm{Sn}(\mathrm{a})$ atoms, as highlighted in Fig. 4, are coordinated by six oxygen atoms. Therefore, they have a similar local environment as in $\mathrm{SnO}_{2}$. The relative positions of the $\mathrm{Sn}-5 s$ and $5 p$ PDOS and their energies with respect to the Fermi energy are analogous to those of the quadrivalent $\mathrm{Sn}$ atom in rutile $\mathrm{SnO}_{2}$. The $\mathrm{Sn}(\mathrm{b})$ in Fig. 4, on the other hand, is analogous to the divalent $\mathrm{Sn}$ atom in $\mathrm{SnO}$. The hybridization of Sn-5s and $5 p$ states can be found at the top of the valence band where O- $2 p$ states also exist. The hybridization is related to the formation of the lone pairs,

TABLE I. Predicted structures of the (101)-layered $\mathrm{Sn}_{3} \mathrm{O}_{4}$ and $\mathrm{Sn}_{2} \mathrm{O}_{3}$ compounds.

\begin{tabular}{lcccccccc}
\hline \hline & Space group & $a(\AA)$ & $b(\AA)$ & $c(\AA)$ & $\alpha$ & $\beta$ & $\gamma$ & Atomic fractional coordinates \\
\hline $\mathrm{Sn}_{3} \mathrm{O}_{4}$ & $P 2_{1} / c$ & 8.21 & 4.93 & 5.85 & 90 & 94.7 & 90 & $\mathrm{Sn}(0.500,0.000,0.500),(0.826,0.000,0.707)$ \\
& & & & & & & & $\mathrm{O}(0.733,0.675,0.503),(0.403,0.698,0.774)$ \\
$\mathrm{Sn}_{2} \mathrm{O}_{3}$ & $P 2_{1} / c$ & 11.12 & 4.89 & 5.84 & 90 & 77.1 & 90 & $\mathrm{Sn}(0.370,0.000,0.329),(0.122,0.997,0.106)$ \\
& & & & & & & & $\mathrm{O}(0.198,0.699,0.858),(0.702,0.677,0.447),(0.050,0.307,0.347)$ \\
\hline \hline
\end{tabular}




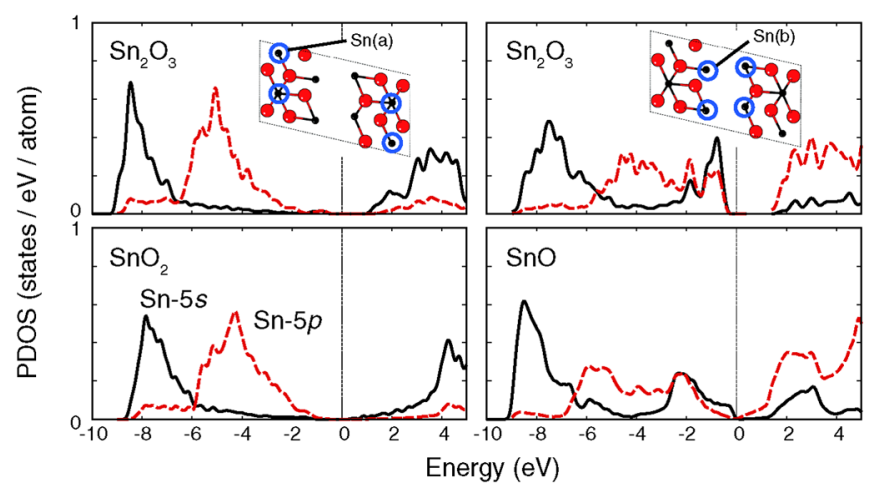

FIG. 4 (color online). PDOS of sixfold-coordinated $\mathrm{Sn}$ (a) and asymmetrically oxygen-coordinated $\mathrm{Sn}(\mathrm{b})$ in the (101)-layered $\mathrm{Sn}_{2} \mathrm{O}_{3}$. The PDOS of $\mathrm{Sn}$ atoms in rutile $\mathrm{SnO}_{2}$ and that of $\mathrm{Sn}$ atoms in litharge $\mathrm{SnO}$ are shown for comparison.

which is the characteristics of the divalent $\mathrm{Sn}$, as discussed for $\mathrm{SnO}$ [32]. These results imply that $\mathrm{Sn}(\mathrm{a})$ and $\mathrm{Sn}(\mathrm{b})$ in $\mathrm{Sn}_{2} \mathrm{O}_{3}$ are quadrivalent and divalent, respectively. No other kinds of Sn atoms are present, which indicates the absence of trivalent $\mathrm{Sn}$ in $\mathrm{Sn}_{2} \mathrm{O}_{3}$. The same conclusion is obtained for $\mathrm{Sn}_{3} \mathrm{O}_{4}$ and the other layered homologous tin oxides. The nonstoichiometric tin oxides thus show a homologous series of $\mathrm{Sn}_{n+1} \mathrm{O}_{2 n}$ in which divalent $\mathrm{Sn}$ atoms are facing each other across the oxygen vacancy layer on the (101) plane. The structures of the homologous series of $\mathrm{Sn}_{n+1} \mathrm{O}_{2 n}$ should originate from the formation of the lone pairs. Atomic structures and electronic mechanism behind the nonstoichiometric structures are markedly different between two rutile-type oxides of quadrivalent cations, i.e., $\mathrm{TiO}_{2}$ and $\mathrm{SnO}_{2}$.

In this study, we investigated the structures and phase stabilities of rutile-type tin oxides, combining firstprinciples calculations with the cluster expansion technique, in particular, those of the controversial $\mathrm{Sn}_{2} \mathrm{O}_{3}$ and $\mathrm{Sn}_{3} \mathrm{O}_{4}$ intermediate compounds. We predicted a homologous series of $\mathrm{Sn}_{n+1} \mathrm{O}_{2 n}$ in which oxygen vacancies are layered on (101) planes. The theoretical structures are consistent with experimental x-ray diffraction profiles. The homologous structures consist of quadrivalent and divalent tin atoms. No trivalent $\mathrm{Sn}$ atoms are formed. The present first-principles prediction can trigger atomic-scale experiments such as hyperfine interaction measurements that can verify the extended-defect structures of $\mathrm{SnO}_{2-x}$.

This study was supported by Program for Improvement of Research Environment for Young Researchers from Special Coordination Funds for Promoting Science and Technology (SCF) commissioned by the Ministry of Education, Culture, Sports, Science and Technology (MEXT) of Japan.

\footnotetext{
*seko@cms.mtl.kyoto-u.ac.jp
}

${ }^{\dagger}$ Present address: Institut für Anorganische Chemie, Rheinisch-Westfälische Technische Hochschule Aachen (RWTH), Landoltweg 1, 52056 Aachen, Germany.

[1] S. Andersson and A. Magnéli, Naturwissenschaften 43, 495 (1956).

[2] S. Andersson, B. Collen, U. Kuylenstierna, and A. Magnéli, Acta Chem. Scand. 11, 1641 (1957).

[3] J. S. Andersson and B. G. Hyde, J. Phys. Chem. Solids 28, 1393 (1967).

[4] G. H. Moh, Chem. Erde 33, 243 (1974).

[5] D. J. McPherson and M. Hanson, Trans. ASME 45, 915 (1953).

[6] S. Cahen, N. David, J.M. Fiorani, A. Maître, and M. Vilasi, Thermochim. Acta 403, 275 (2003).

[7] X. Q. Pan and L. Fu, J. Appl. Phys. 89, 6048 (2001).

[8] M. A. Mäki-Jaskari and T. T. Rantala, Model. Simul. Mater. Sci. Eng. 12, 33 (2004).

[9] F. Gauzzi, Ann. Chim. (Paris) 53, 1503 (1963).

[10] V. G. Murken and M. Trömel, Z. Anorg. Allg. Chem. 397, 117 (1973).

[11] H. Giefers, F. Porsch, and G. Wortmann, Solid State Ionics 176, 199 (2005).

[12] M. S. Moreno, G. Punte, G. Rigotti, R. C. Mercader, A. D. Weisz, and M. A. Blesa, Solid State Ionics 144, 81 (2001).

[13] M.S. Moreno, A. Varela, and L.C. Otero-Díaz, Phys. Rev. B 56, 5186 (1997).

[14] Ç. Kılıç and A.Zunger, Phys. Rev. Lett. 88, 095501 (2002).

[15] J. M. Sanchez, F. Ducastelle, and D. Gratias, Physica (Amsterdam) 128A, 334 (1984).

[16] D. de Fontaine, Solid State Physics (Academic Press, New York, 1994), Vol. 47.

[17] F. Ducastelle, Order and Phase Stability in Alloys (Elsevier Science, New York, 1994).

[18] P. E. Blöchl, Phys. Rev. B 50, 17953 (1994).

[19] J. P. Perdew, K. Burke, and M. Ernzerhof, Phys. Rev. Lett. 77, 3865 (1996).

[20] G. Kresse and J. Hafner, Phys. Rev. B 47, R558 (1993).

[21] G. Kresse and J. Furthmüller, Phys. Rev. B 54, 11169 (1996).

[22] G. Kresse and D. Joubert, Phys. Rev. B 59, 1758 (1999).

[23] G. L. W. Hart, V. Blum, M. J. Walorski, and A. Zunger, Nat. Mater. 4, 391 (2005).

[24] M. Stone, J. R. Stat. Soc. Ser. B Methodol. 36, 111 (1974).

[25] A. van de Walle and G. Ceder, J. Phase Equilib. 23, 348 (2002).

[26] A. Seko, http://sourceforge.net/projects/clupan (2007).

[27] A. Seko, K. Yuge, F. Oba, A. Kuwabara, I. Tanaka, and T. Yamamoto, Phys. Rev. B 73, 094116 (2006).

[28] A. Seko, K. Yuge, F. Oba, A. Kuwabara, and I. Tanaka, Phys. Rev. B 73, 184117 (2006).

[29] S. Kirkpatrick, C.D. Gelatt, and M.P. Vecchi, Science 220, 671 (1983).

[30] S. Kirkpatrick, J. Stat. Phys. 34, 975 (1984).

[31] In both Refs. [9,10], powder samples were obtained by the disproportionation of $\mathrm{SnO}$. In the case of $\mathrm{Sn}_{2} \mathrm{O}_{3}$ (Ref. [10]), the stoichiometry was determined by the weight gain of the sample during the reaction. Some residual XRD peaks of $\mathrm{SnO}_{2}$ were subtracted by the authors of Ref. [10].

[32] A. Walsh and G. W. Watson, Phys. Rev. B 70, 235114 (2004). 